ORIGINAL ARTICLE

\title{
Effect of birth order on neonatal morbidity and mortality among very low birthweight twins: a population based study
}

\author{
E S Shinwell, I Blickstein, A Lusky, B Reichman
}

Arch Dis Child Fetal Neonatal Ed 2004;89:F145-F148. doi: 10.1136/adc.2002.021584

See end of article for authors' affiliations .....................

Correspondence to: Dr Shinwell, Department of Neonatology, Kaplan Medical Center, Rehovot Israel; eric_s@clalit.org.il

Accepted 11 March 2003
Objective: To study the effect of birth order on the risk for respiratory distress syndrome (RDS), chronic lung disease (CLD), adverse neurological findings, and death in very low birthweight (VLBW; $<1500 \mathrm{~g}$ ) twins.

Methods: A population based study of VLBW infants from the Israel National VLBW Infant Database. The sample included all complete sets of VLBW twin pairs admitted to all 28 neonatal intensive care units between 1995 and 1999. Outcome variables were compared by birth order and stratified by mode of delivery and gestational age, using General Estimating Equation models, with results expressed as odds ratio (OR) with $95 \%$ confidence interval (Cl).

Results: Second twins were at increased risk for RDS (OR 1.51, 95\% Cl 1.29 to 1.76), CLD (OR 1.36, 95\% $\mathrm{Cl} 1.11$ to 1.66), and death (OR 1.24,95\% Cl 1.02 to 1.51) but not for adverse neurological findings (OR $1.20,95 \% \mathrm{Cl} 0.91$ to 1.60 ). Mode of delivery did not significantly influence outcome. The odds ratio for RDS in the second twin was inversely related to gestational age, and the increased risk for RDS and CLD was found in both vaginal and caesarean deliveries.

Conclusions: VLBW second twins are at increased risk for acute and chronic lung disease and neonatal mortality, irrespective of mode of delivery.
$\mathrm{P}$ reterm second twins are at increased risk for respiratory distress syndrome (RDS). ${ }^{1-9}$ Although this observation has been recognised for many years, improved obstetric and neonatal care, including antenatal steroids and postnatal surfactant, have improved the outcome of high risk very low birthweight (VLBW; < $1500 \mathrm{~g}$ ) infants, and therefore this observation may no longer be valid. Moreover, previous reports have mostly studied relatively small series of infants.

Accordingly, we evaluated a large population database for the effect of birth order on RDS, chronic lung disease (CLD), adverse neurological findings, and mortality in VLBW twins. As previous studies have related the increased risk of second twins to malpresentation associated asphyxia, this study also examines the interaction of perinatal depression, malpresentation, and gestational age with the effect of birth order on these outcomes.

\section{METHODS}

\section{Israel National VLBW Infant Database}

Infants with birth weight of $1500 \mathrm{~g}$ or less who are born alive in all of the country's 28 neonatal units are included in the database. Data are prospectively collected on a prestructured form and include information on the parents, maternal pregnancy history and antenatal care, mode of delivery, infant's status at birth, procedures and morbidity during hospital stay, and outcome at discharge. Definitions used are concomitant with those of the Vermont-Oxford Trials Network and were defined by the scientific committee before data collection and have remained unaltered since. ${ }^{10}$ Once collected by the local investigators, the data are sent to the database coordinator, checked for missing items and logic errors, corrected, completed, and then entered into a computerised database. Patient information is cross checked with the national birth registry, and any missing data are requested from the birth hospital. Data are collected on all infants until discharge home or death.

\section{Study sample}

For the purposes of this study, we included all sets of twins in which both twins had a birth weight of $1500 \mathrm{~g}$ or less and gestational age of 24 weeks or more. The vast majority ( $86 \%$ ) were of gestational age 32 weeks or less. Sets in which one member did not meet the criteria were excluded. We also excluded 31 cases of caesarean section for the second twin after vaginal delivery of the first twin.

From 1995 through 1999, the database included records of 7047 infants which comprise $99 \%$ of all VLBW live births in Israel (approximately $1.2 \%$ of all births). Thirty percent (2131) of the infants were twins, and 1328 infants (664 twin pairs) fulfilled the study criteria. In the subsequent analysis of major neonatal morbidities, further exclusions were due to neonatal deaths of one or both members of the twin pair, or missing data. The analysis of RDS included 651 pairs after exclusion of 13 delivery room deaths. CLD was evaluated in 434 pairs, excluding neonatal deaths or missing data $(\mathrm{n}=$ 24) related to oxygen therapy at 28 days of age. Adverse neurological findings were considered in 412 pairs, excluding twin pairs in whom one or both infants died or an appropriate ultrasound was not performed $(\mathrm{n}=46)$. The minimum requirement for ultrasound was two examinations, one before age 28 days for identification of intraventricular haemorrhage and one after age 28 days for periventricular leucomalacia and ventricular dilatation.

\section{Definition of outcome variables}

Death was defined as occurring during the hospital stay. Delivery room resuscitation included endotracheal intubation and/or cardiac massage or adrenaline (epinephrine) administration. RDS was defined by characteristic clinical and radiographic findings. CLD was defined as a clinical diagnosis

Abbreviations: CLD, chronic lung disease; RDS, respiratory distress syndrome; VLBW, very low birthweight 
of bronchopulmonary dysplasia and requirement for supplemental oxygen therapy at 28 days of life. Adverse neurological findings were defined as one or more of the following: grade 3 or 4 periventricular-intraventricular haemorrhage, cystic periventricular leucomalacia, or post-haemorrhagic hydrocephalus. ${ }^{11}$

\section{Statistical analysis}

For each outcome variable, we used the Generalised Estimating Equation approach to estimate and test the odds ratios for first versus second twins. ${ }^{12}$ In a univariate analysis, the odds ratio estimate using this method is equal to the usual estimate of odds of the outcome among second twins divided by the estimate of odds of outcome among first twins. We chose this estimate as it included the data from all sets of twins and not only the discordant sets. The Generalised Estimating Equation method takes into account the correlation between the outcomes of sibling pairs.

To test if the odds for second versus first twins ratios were different for twins delivered vaginally compared with caesarean section and by groups of gestational age, stratified analysis was performed by mode of delivery and gestational age. Results are presented as odds ratio (OR) and 95\% confidence interval (CI). Analyses were performed using the SAS (SAS Institute, Inc, Cary, North Carolina, USA) statistical software (version 8.2).

\section{RESULTS}

\section{Characterisation of study sample}

The study population comprised 664 VLBW twin pairs. The mean (SD) gestational age at delivery was 28.4 (2.4) weeks, and the birth weights of the first and second twins were similar (1072 (274) v 1056 (270) g). Antenatal steroids were administered in $70 \%$, and $65 \%$ were delivered by caesarean section.

\section{Outcome variables}

The incidence of the outcome variables in the study sample was RDS 70\%, CLD 19\%, adverse neurological findings 39\%, and mortality $21 \%$. Table 1 shows the comparison of the outcome variables between the first and second twins. Second twins were at significantly higher risk for RDS (OR $1.51,95 \%$ CI 1.29 to 1.76 ), CLD (OR 1.36, 95\% CI 1.11 to 1.66 ), and death (OR $1.24,95 \%$ CI 1.02 to 1.51 ). No significant difference was found between the groups of twins in the risk for adverse neurological findings (OR 1.20, 95\% CI 0.91 to 1.60 ). Most twin pairs were concordant for each of the outcome variables (death $81 \%$, RDS $82 \%$, CLD $88 \%$, adverse neurological findings 79\%).

Table 2 shows similar odds ratios for each of the outcomes in twin pairs born vaginally or by caesarean section. The excess risk in second twins for RDS and CLD was significant in infants delivered by caesarean section or vaginally. However, as the sample in this stratified analysis was considerably smaller, the odds ratio for mortality in these subgroups did not quite meet statistical significance.

\section{Perinatal status}

Second twins were at higher risk for malpresentation (OR $2.10,95 \%$ CI 1.71 to 2.57 ). However, no difference was found between the twin groups in Apgar scores at five minutes (OR $1.05,95 \%$ CI 0.86 to 1.28 ) or in the need for resuscitative measures in the delivery room (OR $1.08,95 \%$ CI 0.95 to 1.22 ).

\section{Gestational age}

To assess the risk for RDS and CLD while controlling for gestational age, we calculated odds ratios for these outcome variables in subgroups of 24-27 weeks, 28-30 weeks, and greater than 30 weeks (table 3 ). The increased risk for RDS in second twins appeared to be inversely related to gestational age, with maximum effect in the 24-27 week group. The increased risk for CLD was found to be significant only in the 24-27 week gestation group. The other outcomes were not compared by gestational age because of low incidence.

\section{DISCUSSION}

This study confirms that VLBW second twins are at significantly increased risk for RDS, CLD, and death and that the excess risk for RDS and CLD is most pronounced in infants born at 24-27 weeks gestation. In addition, it is of note that delivery by caesarean section does not appear to protect the second twin from this increased risk.

This study has certain strengths and weaknesses that may influence the interpretation of the findings. The major strength is the large, population based sample of high risk VLBW neonates, drawn from both local general and university teaching hospitals. The focus on VLBW infants increased the likelihood of finding real differences between the twin groups, and further sensitivity was achieved by the use of within pairs statistical analyses compared with the non-paired approach used in the studies of Chen et $a l^{13}$ and Neilsen et al. ${ }^{14}$

However, a number of limitations need to be discussed. Firstly, although the sample was large, it was not large enough to resolve the issue of the influence of gestational age on the increased risk for RDS in second twins. Hacking et al found a direct correlation with gestational age, whereas this study, with a larger sample, found an inverse correlation. To study this question more satisfactorily, a much larger sample will be required. Another concern is the definition of CLD as a clinical diagnosis of bronchopulmonary dysplasia and requirement for supplemental oxygen therapy at 28 days of life. The studies of Hacking et al ${ }^{8}$ and Donovan et al both found second twins to be at increased risk for RDS but not for CLD, when defined as oxygen requirement at 36 weeks gestational age. The shorter term definition was used in our study because of the higher availability of the information in the database. Clearly, as the incidence of oxygen dependency is lower at 36 weeks, there is less likelihood of finding significant intertwin differences or, alternatively, there may be a real difference at 28 days which is diminished by 36 weeks. A larger study with reliable data at both time points will be required to resolve this.

Table 1 Incidence, odds ratios, and 95\% confidence intervals for outcome variables in first and second twins

\begin{tabular}{llllll}
\hline Variable & No of twin pairs & First twin (\%) & Second twin (\%) & OR & $95 \% \mathbf{C l}$ \\
\hline Death & 664 & 19.6 & 23.2 & 1.24 & 1.02 to 1.51 \\
RDS & 651 & 65.7 & 74.3 & 1.51 & 1.29 to 1.76 \\
CLD & 434 & 18.4 & 23.5 & 1.36 & 1.11 to 1.66 \\
Neuro & 412 & 18.2 & 21.1 & 1.20 & 0.91 to 1.60 \\
\hline
\end{tabular}

RDS, Respiratory distress syndrome; CLD, chronic lung disease; Neuro, adverse neurological findings; OR, odds ratio; $95 \% \mathrm{Cl}, 95 \%$ confidence interval. 
Table 2 Odds ratios and $95 \%$ confidence intervals for outcome variables in first and second twins delivered vaginally or by caesarean section

\begin{tabular}{llllll}
\hline & Vaginal delivery & & & \multicolumn{2}{l}{ Caesarean section } \\
\cline { 2 - 3 } \cline { 5 - 6 } \cline { 5 - 6 } & No of twin pairs & OR $(95 \% \mathrm{Cl})$ & & No of twin pairs & OR $(95 \% \mathrm{CI})$ \\
\hline Death & 233 & $1.33(0.72$ to 2.46$)$ & & 431 & $1.53(0.99$ to 2.36$)$ \\
RDS & 227 & $1.39(1.11$ to 1.72$)$ & & 424 & $1.58(1.29$ to 1.94$)$ \\
CLD & 142 & $1.45(1.03$ to 2.04$)$ & & 292 & $1.33(1.04$ to 1.70$)$ \\
Neuro & 131 & $1.19(0.76$ to 1.86$)$ & 281 & $1.21(0.84$ to 1.74$)$ \\
\hline
\end{tabular}

RDS, Respiratory distress syndrome; CLD, chronic lung disease; Neuro, adverse neurological findings; OR, odds ratio; $95 \% \mathrm{Cl}, 95 \%$ confidence interval.

Table 3 Effect of gestational age on odds ratios for respiratory distress syndrome (RDS) and chronic lung disease (CLD) in first and second twins

\begin{tabular}{|c|c|c|c|c|c|c|}
\hline \multirow[b]{2}{*}{ GA (weeks) } & \multicolumn{3}{|l|}{ RDS } & \multicolumn{3}{|l|}{ CLD } \\
\hline & $\mathrm{n}$ & OR & $95 \% \mathrm{Cl}$ & $\mathrm{n}$ & OR & $95 \% \mathrm{Cl}$ \\
\hline $24-27$ & 216 & 2.48 & 1.31 to 4.69 & 84 & 1.67 & 1.10 to 2.54 \\
\hline $28-30$ & 304 & 1.71 & 1.34 to 2.19 & 233 & 1.43 & 0.97 to 2.09 \\
\hline $31+$ & 131 & 1.42 & 0.95 to 2.12 & 117 & 1.53 & 0.55 to 4.24 \\
\hline
\end{tabular}

The influence of birth order on the other outcome variables remains less clear. We found a small but significant increase in mortality risk (OR $1.24,95 \%$ CI 1.02 to 1.51 ), which was not found in the studies of VLBW infants ${ }^{8} 9$ and of all preterm infants. ${ }^{15}$ None of the population based studies found an increase in intraventricular haemorrhage or adverse neurological findings.

Previous studies have suggested that the increased risk for RDS in non-presenting twins is only found in infants delivered vaginally and that this relates to the increased incidence of malpresentation and presumed "asphyxia" in the second twin. ${ }^{7}$ This and other studies did not confirm such findings. ${ }^{16}$ Although second twins had increased risk for malpresentation, there was no increased risk for low five minute Apgar scores or need for resuscitative measures. It follows that clinically evident asphyxia is not part of the cause of the increased risk of second twins for RDS. However, an untested possibility is that transient hypoxia and/or acidosis may affect surfactant function, which may contribute to the development of mild respiratory distress. ${ }^{17}$

In summary, we conclude that, even in the era of widespread use of antenatal steroids, postnatal surfactant, and modern intensive care, VLBW second twins appear to have an increased risk for acute and chronic lung disease and death. The mechanisms of this effect remain partially understood.

\section{ACKNOWLEDGEMENTS}

The Israel National Very Low Birth Weight Infant Database is supported by the Israel Center for Disease Control and the Ministry of Health.

The Israel Neonatal Network participating centres (by alphabetical order) are: Assaf Harofeh Medical Center, Rishon Le Zion

Barzilay Medical Center, Ashkelon

Bikur Holim Hospital, Jerusalem

Bnei Zion Medical Center, Haifa

Carmel Medical Center, Haifa

English (Scottish) Hospital, Nazareth

French Hospital, Nazareth

Hadassah University Hospital, Ein-Kerem, Jerusalem

Hadassah University Hospital, Har Hatzofim, Jerusalem

Haemek Medical Center, Afula

Hillel Yaffe Medical Center, Hadera

Italian Hospital, Nazareth

Kaplan Medical Center, Rehovot
Laniado Hospital, Netanya

Mayanei Hayeshua Hospital, Bnei Brak

Meir Medical Center, Kfar Saba

Misgav Ladach Hospital, Jerusalem

Nahariya Hospital, Nahariya

Poria Hospital, Tiberias

Rambam Medical Center, Haifa

Rivka Ziv Hospital, Tsfat

Schneider Children's Medical Center of Israel and Rabin Medical Center (Beilinson Campus), Petach Tikva

Shaarei Zedek Hospital, Jerusalem

Sheba Medical Center, Tel Hashomer

Soroka Medical Center, Beersheva

Sourasky Medical Center, Tel Aviv

Wolfson Medical Center, Holon

Yoseftal Hospital, Eilat

Coordinating centre: Women and Children's Health Research Unit, Gertner Institute, Tel Hashomer

\section{Authors' affiliations}

E S Shinwell, Department of Neonatology, Kaplan Medical Center, Rehovot, and Hadassah Medical School, Hebrew University, Jerusalem Israel

I Blickstein, Department of Obstetrics and Gynecology, Kaplan Medical Center, Rehovot, and Hadassah Medical School, Hebrew University, Jerusalem Israel

A Lusky, B Reichman, Women and Children's Health Research Unit, Gertner Institute, Sheba Medical Center, Tel Hashomer, Israel

B Reichman, Sackler School of Medicine, Tel Aviv University, Tel Aviv, Israel

\section{REFERENCES}

1 Ellis RF, Berger GS, Keith L, et al. The Northwestern University Multihospital Twin Study. II. Mortality of first versus second twins. Acta Genet Med Gemellol (Roma) 1979;28:347-52.

2 Lankenau HM. A genetic and statistical study of the respiratory distress syndrome. Eur J Pediatr 1976;123:167-77.

3 Rokos J, Vaesuorn O, Nachman R, et al. Hyaline membrane disease in twins. Pediatrics 1968:42:204-5.

4 Verduzco RD, Rosario R, Rigatto $H$. Hyaline membrane disease in twins: a 7 year review with a study on zygosity. Am J Obstet Gynecol 1976;125:668-71.

5 Neligan G, Robson E, Hey E. Hyaline membrane disease in twins. Pediatrics 1969;43:143.

6 Burkett G, Baver CR, Morrison JM, et al. Effect of prenatal dexamethasone administration on prevention of respiratory distress syndrome in twin pregnancies. J Perinatol 1986;6:304-8. 
7 Arnold C, McLean FH, Kramer MS, et al. Respiratory distress syndrome in second-born versus first-born twins. A matched case-control analysis. N Engl J Med 1987;317:1121-5.

8 Hacking D, Watkins A, Fraser S, et al. Respiratory distress syndrome and birth order in premature twins. Arch Dis Child Fetal Neonatal Ed 2001;84:F117-21.

9 Donovan EF, Ehrenkrantz RA, Shankaran S, et al. Outcomes of very low birth weight twins cared for in the National Institute of Child Health and Human Development Neonatal Research Network's intensive care units. Am J Obstet Gynecol 1998;179:742-9.

10 Vermont-Oxford trials network database project. Manual of operations. Burlington, VT: Vermont-Oxford trials network, 1993, release 2.0

11 Papille LA, Burstein J, Burstein R, et al. Incidence and evolution of subependymal and intraventricular hemorrhage: a study of infants with birth weights less than 1500 grams. J Pediatr 1978;92:529-34.
12 Zeger SL, Liang K. Longitudinal data analysis for discrete and continuous outcome. Biometrics 1986;42:121-30.

13 Chen SJ, Vohr BR, Oh W. Effects of birth order, gender and intrauterine growth retardation on the outcome of very low birth weight in twins. J Pediatr 1993;123:132-6.

14 Nielsen HC, Harvey-Wilkes K, MacKinnon B, et al. Neonatal outcome of very premature infants from multiple and singleton gestations. Am J Obstet Gynecol 1997; 177:653-9.

15 Smith GCS, Pell JP, Dobbie R. Birth order, gestational age, and risk of delivery related perinatal death in twins: retrospective cohort study. $B M J$ 2002;325: 1004

16 Prins RP. The second-born twin: can we improve outcomes? Am J Obstet Gynecol 1994; 170:1649-56.

17 Fanaroff AA, Martin RJ. Neonatal-perinatal medicine. In: Diseases of the fetus and infant, 6th edn. St Louis, MO: Mosby, 1997:1021. 